\title{
Vitamin D supplementation in early childhood and risk of type 1 diabetes: a systematic review and meta-analysis
}

\author{
C S Zipitis, ${ }^{1}$ A K Akobeng ${ }^{2}$
}

${ }^{1}$ Department of Paediatrics, Stockport NHS Foundation Trust, Stockport, UK; ${ }^{2}$ Department of Paediatric Gastroenterology, Central Manchester and Manchester Children's University Hospitals, Booth Hall Children's Hospital, Manchester, UK

Correspondence to: C S Zipitis, Department of Paediatrics, Stockport NHS Foundation Trust, Poplar Grove, Stockport SK2 7JE, UK; czipitis@hotmail.com

Accepted 17 January 2008 Published Online First 13 March 2008

\begin{abstract}
Objectives: To assess whether vitamin D supplementation in infancy reduces the risk of type 1 diabetes in later life. Methods: This was a systematic review and metaanalysis using Medline, Embase, Cinahl, Cochrane Central Register of Controlled Trials and reference lists of retrieved articles. The main outcome measure was development of type 1 diabetes. Controlled trials and observational studies that had assessed the effect of vitamin $D$ supplementation on risk of developing type 1 diabetes were included in the analysis.
\end{abstract}

Results: Five observational studies (four case-control studies and one cohort study) met the inclusion criteria; no randomised controlled trials were found. Meta-analysis of data from the case-control studies showed that the risk of type 1 diabetes was significantly reduced in infants who were supplemented with vitamin D compared to those who were not supplemented (pooled odds ratio $0.71,95 \% \mathrm{Cl} 0.60$ to 0.84$)$. The result of the cohort study was in agreement with that of the meta-analysis. There was also some evidence of a dose-response effect, with those using higher amounts of vitamin $D$ being at lower risk of developing type 1 diabetes. Finally, there was a suggestion that the timing of supplementation might also be important for the subsequent development of type 1 diabetes.

Conclusion: Vitamin D supplementation in early childhood may offer protection against the development of type 1 diabetes. The evidence for this is based on observational studies. Adequately powered, randomised controlled trials with long periods of follow-up are needed to establish causality and the best formulation, dose, duration and period of supplementation.

Type 1 diabetes is characterised by autoimmune destruction of insulin-producing $\beta$ cells in the pancreas. The specific factors that initiate the autoimmune process are not yet well understood, but $\beta$ cell destruction often begins during infancy and continues over many months or years. ${ }^{1}$ By the time type 1 diabetes is diagnosed, about $80 \%$ of the $\beta$ cells have been destroyed. ${ }^{2}$ Peak incidence occurs around puberty, and the disease is usually diagnosed before age $30 .^{3}$ It is commonest in people of European descent and affects 2 million people in Europe and North America. There is a marked geographic variation in incidence, with a child in Finland being about 400 times more likely than a child in Venezuela to acquire the disease. ${ }^{4}$ It is estimated that currently the incidence is increasing by $3 \%$ per year. ${ }^{5}$ Furthermore, it is predicted that by 2010 the incidence of type I diabetes will be $40 \%$ higher than it was a decade earlier. ${ }^{6}$
The fact that people with affected first-degree relatives are a lot more likely to develop type 1 diabetes than the general population points to an important genetic influence. ${ }^{7}$ However, low concordance among identical twins and the fact that many children with a genetic predisposition to the disease do not develop it suggest that environmental factors are also important. ${ }^{8}$ One of the environmental factors thought to be protective against the development of type I diabetes, is early supplementation with vitamin $\mathrm{D}$.

Vitamin D is either produced endogenously, through skin exposure to sunlight, or exogenously from ingestion of foods and supplements. Breast milk contains little vitamin $\mathrm{D}$, although this is influenced by the vitamin D status of the mother," and this is the reason behind the recommendation for an administered supplement for breastfed infants. ${ }^{10}$ Furthermore, in northern areas, including the northern United States, Canada and most of Europe, little or no vitamin $\mathrm{D}$ is produced in the skin during winter months. ${ }^{11}$ Even in the summer and at lower latitudes, many infants are so thoroughly protected from sun exposure that they produce little endogenous vitamin $\mathrm{D}$. In addition, there is evidence that over the last few decades the uptake $^{12}$ as well as the dosage $\mathrm{e}^{13}$ of vitamin D supplementation have been declining leading to a resurgence of rickets and hypocalcaemia, ${ }^{14}$ as well as speculation about the possible role of vitamin $\mathrm{D}$ in the increasing incidence of type 1 diabetes and other autoimmune conditions such as rheumatoid arthritis ${ }^{15}$ and multiple sclerosis. ${ }^{16}$

Evidence for a causal relationship between vitamin $\mathrm{D}$ supplementation and decreased risk of type 1 diabetes comes from experiments in the non-obese diabetic mouse. ${ }^{3}$ Furthermore, there is evidence of lower plasma 25-hydroxyvitamin D levels at diagnosis of type 1 diabetes compared to controls. ${ }^{17}$ Moreover, epidemiological evidence suggests that type 1 diabetes is more prevalent in higher latitudes of the tropics and subtropics ${ }^{18}$ and that there is a seasonal variation in type 1 diabetes with the largest proportion of cases diagnosed during autumn and winter and the lowest during the summer. ${ }^{19}$ In addition, epidemiological studies ${ }^{20}$ suggest that supplementation with vitamin $\mathrm{D}$ in infants might be important in conferring protection against the development of type 1 diabetes.

In this study, we sought to explore the potential association between vitamin D supplementation in early childhood and reduced risk of type 1 diabetes by conducting a systematic review and metaanalysis of human trials and observational studies. 
Figure 1 Flow chart of studies through the review process.

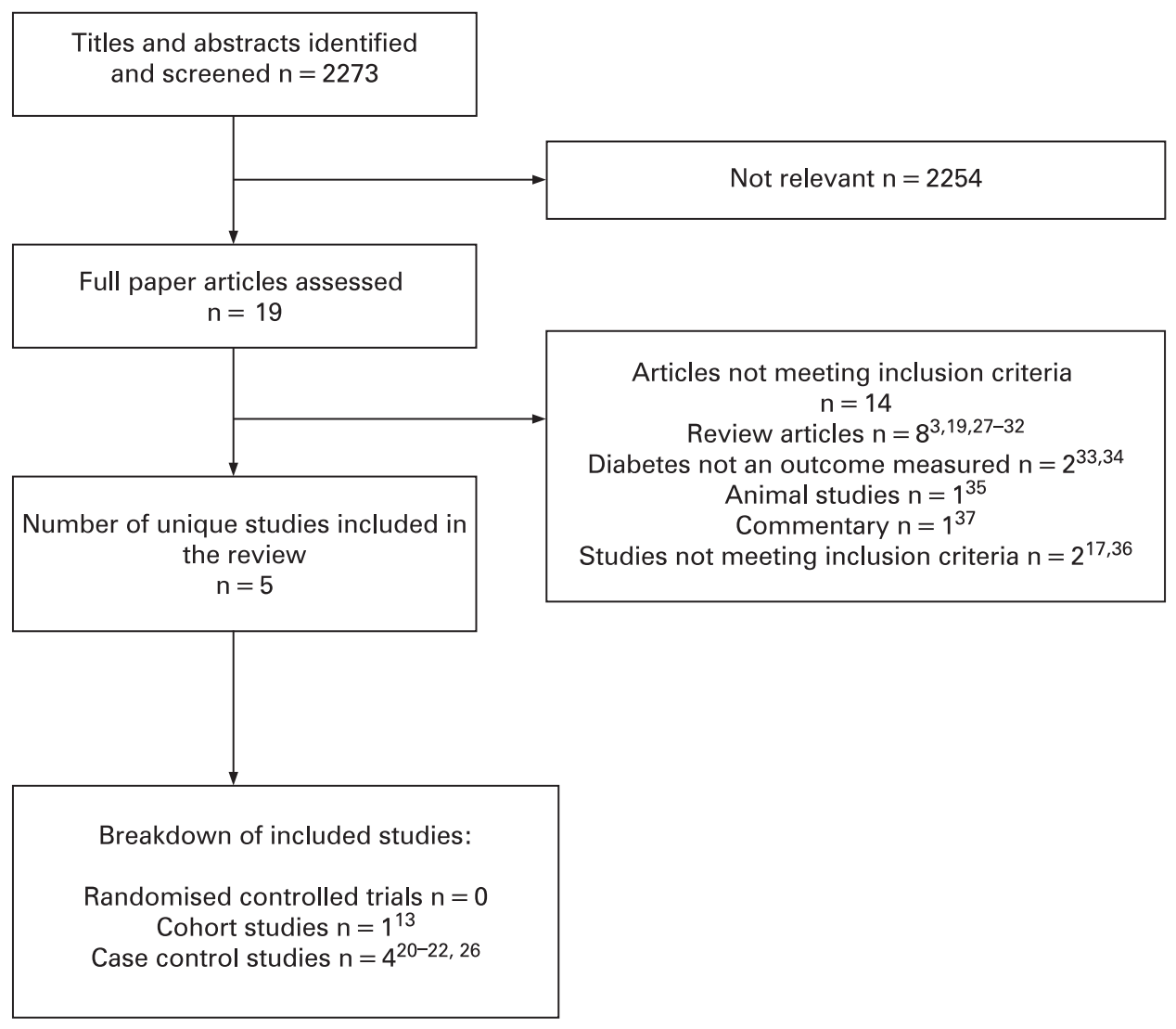

\section{METHODS}

\section{Types of studies}

We searched for randomised controlled trials and observational studies. We included observational studies if they: (1) compared risk of type 1 diabetes in people who were supplemented with vitamin $\mathrm{D}$ with risk in those who were not supplemented, (2) had controlled for potential confounders by matching in the study design or had used risk adjustment in the analysis and (3) had provided sufficient data to allow the reconstruction of 2 by 2 tables or to determine relative risks (RR) or odds ratios (OR) with $95 \%$ confidence intervals ( $95 \%$ CI). Subjects given cod liver oil were considered to have been supplemented with vitamin $\mathrm{D}$ in line with previous publications. ${ }^{13} 2122$

\section{Search strategy}

We systematically searched Medline (1966-June 2007), Embase (1980-June 2007), Cinahl (1982-June 2007) and the Cochrane Central Register of Controlled Trials (issue 2, 2007) for both English and non-English language articles by entering "vitamin D", "vitamin D2", "vitamin D3", "ergocalciferol", "alfacalcidol", "alphacalcidol", "hydroxycholecalciferol", "calcitriol", "dihydroxycholecalciferol", "colecalciferol", "cholecalciferol" and "diabetes". No limits were used.

We then searched the reference lists of all relevant articles retrieved from the computerised database search to find other potentially relevant articles. The titles and/or abstracts of all identified studies were reviewed and full manuscripts obtained for those that appeared potentially relevant.

\section{Assessment of study eligibility}

Each article was independently assessed by two reviewers for eligibility using the inclusion criteria above. Any disagreement among reviewers was discussed and agreement reached by consensus.

\section{Assessment of methodological quality}

Two investigators independently rated the methodological quality of selected studies using the Critical Appraisal Skills Programme (PHRU, Oxford, UK) tool for case-control studies. ${ }^{23}$ We recorded each quality assessment criterion as being "met", "unmet" or "unclear". However, as several criteria were used to assess validity, these were summarised to derive an overall assessment of how valid the results of each study were by grading them as A (low risk of bias), B (moderate risk of bias) or $\mathrm{C}$ (high risk of bias) according to published criteria. ${ }^{24}$

\section{Data extraction}

A data abstraction form was developed and used to extract information on the relevant features and results of included studies. Two reviewers independently extracted and recorded data using a predefined checklist. When data were missing or unclear in a paper, attempts were made to contact the authors for more information.

\section{Statistical methods}

Statistical analyses were performed using StatsDirect statistical software (v 2.6.1; StatsDirect, Altrincham, UK). Individual OR and their $95 \%$ CI from each case-control study were calculated. Where possible, a pooled OR with $95 \%$ CI was calculated. The metaanalysis was conducted using the Mantel-Haenszel method. ${ }^{25}$

\section{Heterogeneity}

The statistical validity of combining the results of the various trials was assessed by examining the homogeneity of the 
Table 1 Summary of methodology of case-control studies

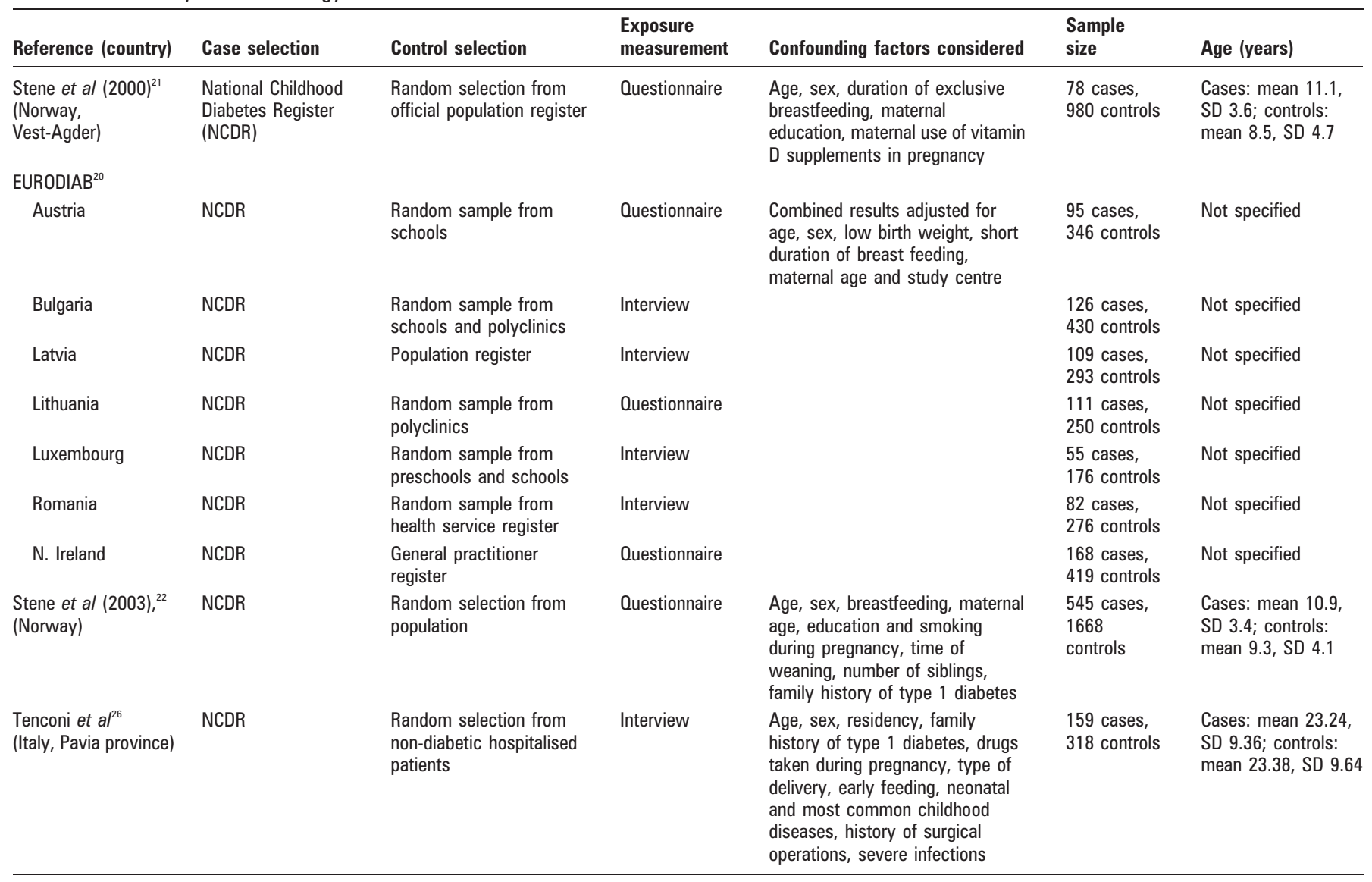

outcomes from the various trials. This was carried out by: (1) using the Cochran $\mathrm{Q}$ test and (2) inspection of the graphical display.

\section{RESULTS}

We identified 19 potentially relevant articles ${ }^{3} 1^{17}{ }^{19-22} 26-37$ on the association between vitamin $\mathrm{D}$ supplementation and the development of type 1 diabetes (fig 1). Five studies were identified that satisfied the inclusion criteria and these were included in the review. ${ }^{1320-22}{ }^{26}$ Four of them were case-control studies $^{20-2226}$ and one was a cohort study. ${ }^{13}$ No randomised control trials were identified. The study by Littorin et $a l^{17}$ was excluded because it did not compare rates in those exposed to or not supplemented with vitamin $\mathrm{D}$, but rather the levels of vitamin $\mathrm{D}$ over time in those with type 1 diabetes. The study by Visalli et $a^{36}$ was excluded because it did not provide enough data for construction of a 2 by 2 table to determine OR and repeated attempts to get more information from the authors proved unsuccessful.

\section{Methodological quality of included studies}

Due to the retrospective design, all the case-control studies were prone to recall bias. All of the included case-control studies except that by Tenconi et $a^{26}$ used healthy children whose nondiabetic status was not specifically confirmed. However, as type 1 diabetes is unlikely to be asymptomatic for a long period of time, the possibility of misclassification for some controls is small. Most case-control studies used cases up to 15 years of age, ${ }^{20-22}$ but Tenconi et $a^{26}$ used a higher cut-off value (30 years). None of the case-control studies included used an objective method to ascertain the vitamin D status of cases or controls, and none attempted to quantify the total amount of vitamin $\mathrm{D}$ intake from the diet or that from exposure to the sun. In addition, none of the studies looked at the ethnic backgrounds of cases versus controls. A summary of the methodology of the included case-control studies is shown in table 1.

The methodological quality of each study was summarised using the categories described above. All case-control studies included were graded $\mathrm{B}$. The cohort study ${ }^{13}$ was graded $\mathrm{B}$ because it did not use an objective method of assessing the vitamin $\mathrm{D}$ status of cases and controls and because no mention of blinding to the outcomes was mentioned. Furthermore, although a fair number of confounding factors were accounted for, some important ones were not, for example duration of breastfeeding and age at weaning.

\section{Association between vitamin D supplementation and type 1 diabetes}

Ever supplemented versus never supplemented

Three of the included case-control studies ${ }^{20}{ }^{21}$ provided enough data on the risk of developing type 1 diabetes in infants who were supplemented with vitamin $D$ versus those not being supplemented, to allow a meta-analysis to be performed on this variable. After contacting the authors of the final case-control study, ${ }^{22}$ enough information was provided to allow inclusion of this study in the meta-analysis. For the EURODIAB study ${ }^{20}$ we followed the example of the authors and used each centre as a separate entry to account for the heterogeneity between centres and also to aid in the assessment of heterogeneity in the global sample. The total number of participants in included studies was 6455 (1429 cases and 5026 controls). In the main analysis using the fixed effects model (fig 2), the risk of developing type 
1 diabetes was significantly reduced in participants who were supplemented with vitamin D (OR $0.71,95 \%$ CI 0.60 to 0.84 ). The results of these studies appeared to be reasonably homogeneous and the test for heterogeneity was negative $(\mathrm{p}=0.13)$.

The result of the meta-analysis is in agreement with the main outcome from the cohort study. ${ }^{13}$ In the latter study, the rate risk for regular versus no supplementation was 0.12 (95\% CI 0.03 to 0.51 ) and for irregular versus no supplementation 0.16 (95\% CI 0.04 to 0.74 ).

\section{Type of supplement used}

There were not enough data in the primary studies to allow comparison of the groups based on type of supplement used.

\section{Dosage of supplementation}

In the majority of studies no information was given about the dosage of supplements used. Stene et al (2003) 22 speculated that the cod liver oil and the different vitamin $\mathrm{D}$ supplements used in Norway at the time in question would contain $10 \mu \mathrm{g}$ of vitamin D. They divided their patients into those who had taken cod liver oil and those who had "other" forms of vitamin D. Concentrating on the group that had the cod liver oil, one can see that as the frequency of supplementation increases from one to four times per week to more than five times per week, the OR of developing type 1 diabetes decreases (OR 0.81, 95\% CI 0.55 to 1.19 and OR $0.74,95 \%$ CI 0.56 to 0.99 , respectively). There is a negligible change in OR in those that had the "other" types of vitamin D supplement (OR 0.99, 95\% CI 0.69 to 1.42 and OR $0.97,95 \%$ CI 0.73 to 1.29 , respectively).

There is evidence from the Hypponen study ${ }^{13}$ that those who used the recommended dose of 2000 IU regularly had an RR of 0.22 (95\% CI 0.05 to 0.89 ) compared with those who regularly used less than the recommended dose. Furthermore, the authors showed that with an increase in regularity of supplementation as well as dose used, the incidence of type 1 diabetes tended to decrease. ${ }^{13}$ Finally, they reported that those with suspected rickets had an increased risk of developing type 1 diabetes, although the result was not statistically significant (RR 3.0, 95\% CI 1.0 to 9.0$)$.

\section{Duration of supplementation}

Only the EURODIAB study ${ }^{20}$ considered duration of supplementation as a factor. Although there was some improvement in the OR, they did not find any significant difference between those who were supplemented for less than a year (OR 0.69, $95 \%$ CI 0.52 to 0.93 ) and those who were supplemented for more than a year (OR $0.64,95 \%$ CI 0.47 to 0.89 ).

\section{Timing of supplementation}

The report by Stene et al (2003) $)^{22}$ is the only one of the identified studies that looked at the effect of the time of starting supplementation with vitamin $\mathrm{D}$. It appears that those who had cod liver oil between 7 and 12 months of age had lower chances of developing type 1 diabetes in later life compared to those who were supplemented between 0 and 6 months of age (OR 0.55, $95 \%$ CI 0.31 to 0.96 and OR $0.80,95 \%$ CI 0.61 to 1.06 , respectively). A similar, albeit less impressive, result was obtained for the "other" vitamin D supplements (OR 0.98, $95 \%$ CI 0.65 to 1.49 and OR $1.02,95 \%$ CI 0.77 to 1.35 , respectively).

\section{DISCUSSION}

The results of this study suggest that vitamin D supplementation in infancy may offer protection against the development of type 1 diabetes. Meta-analysis of data from four studies, which included children from many different European countries, indicated that children being supplemented had a $29 \%$ reduction in risk of developing type 1 diabetes compared with their peers who were not being supplemented. The reduction in risk was also demonstrated in a cohort study. ${ }^{13}$ The study by Stene et al $(2003)^{22}$ reported no association between vitamin D supplementation and subsequent diabetes development. Although it was not clear in the paper how many of these patients used both vitamin $\mathrm{D}$ and cod liver oil and how many used neither of the two, we managed to obtain this information by contacting

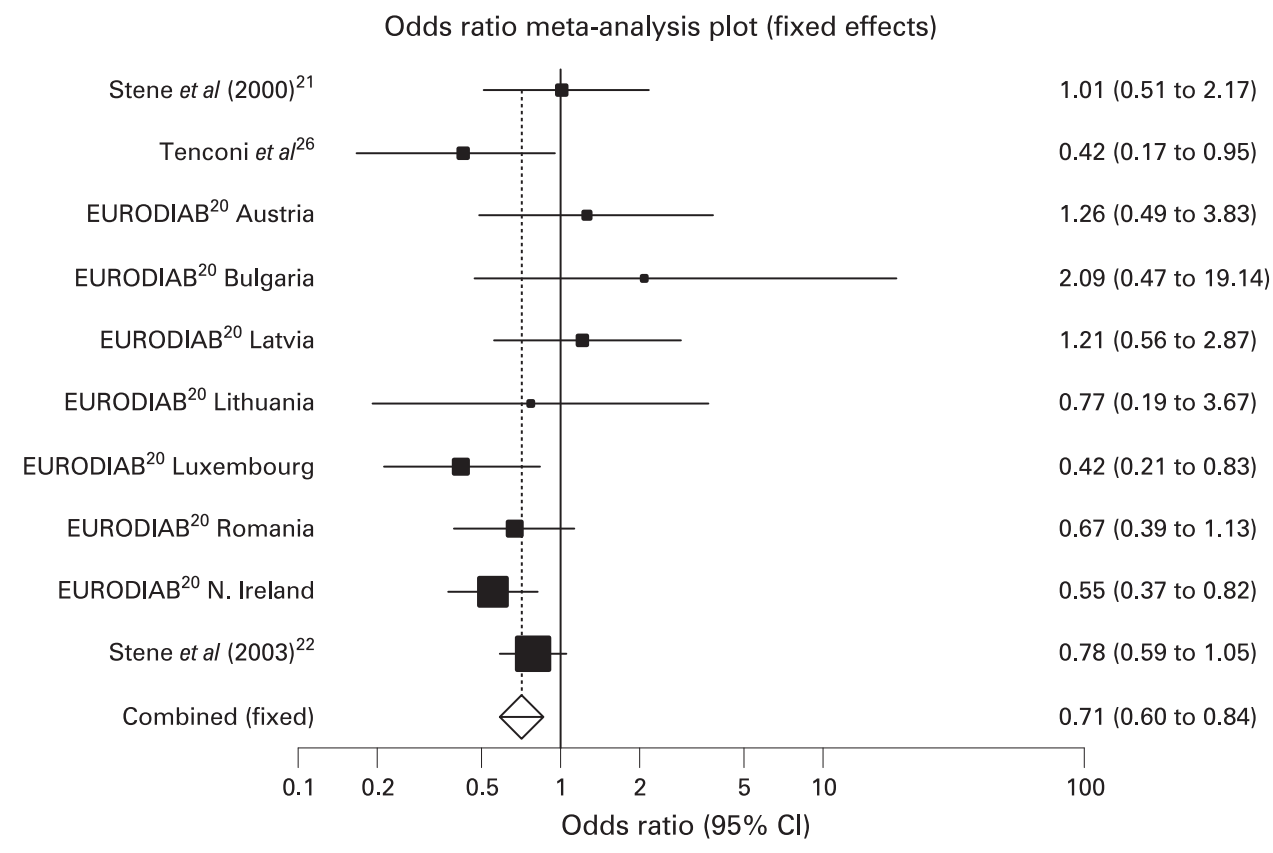

Figure 2 Odds ratios $(95 \% \mathrm{Cl})$ of effect of vitamin D supplementation in infancy on development of type 1 diabetes. 
the authors, something that allowed inclusion of the study in the meta-analysis. Some studies were not able to show an association with a reduction in risk of type 1 diabetes, ${ }^{21}{ }^{36}$ but none of them were associated with an increased risk.

Furthermore, there is evidence of a dose-response effect. The cohort study ${ }^{13}$ showed that those who had rickets diagnosed earlier in life (and were thus more likely to be those with the lowest amounts of vitamin D) were more likely to develop type 1 diabetes. In addition, those that were supplemented more regularly or had higher doses of vitamin D supplements, displayed a reduced risk of developing type 1 diabetes. The positive findings with increasing frequency of use were also confirmed in one of the case-control studies ${ }^{22}$ that looked at this variable.

One of the included studies ${ }^{22}$ suggests that supplementation from 7 to 12 months of age is more beneficial than supplementation from birth to 6 months. However, the former could be a marker of longer supplementation. At the same time, infants of mothers who are themselves vitamin $\mathrm{D}$ deficient would be at an increased risk of hypocalcaemic complications in the first few months of life,,$^{14}$ something that would necessitate earlier supplementation. Finally, there are many unexplored variables including an overall lack of accurate, reliable feeding information which could explain this observation.

The exact mechanism by which vitamin $\mathrm{D}$ supplementation protects against type 1 diabetes is unclear, but it has been suggested that this is likely to be through the prevention of hypovitaminosis $\mathrm{D}^{27}$ The identification of receptors for the active form of vitamin $D$ in both $\beta$ cells and immune cells ${ }^{27}$ has led to a number of studies for the delineation of these pathways. There is evidence for a physiological role for vitamin $\mathrm{D}$ in the immune system, and also for a protective effect of the vitamin from cytokine-induced $\beta$ cell dysfunction. ${ }^{27}$

\section{Strengths and weaknesses of the study}

Our review included studies from many European countries and included four case-control studies and a cohort study. We searched multiple databases and reference lists to minimise the chance of missing relevant studies. We minimised subjectivity by carrying out study selection, data extraction and quality assessment in duplicate. However, the validity of the results of a systematic review depends on the validity of the included studies. Many of the included studies failed to take all the steps necessary to avoid bias. Our conclusions were, therefore, limited by the quality of included studies and the information provided.

The included case-control studies are subject to recall bias. If, for instance, parents of children with type 1 diabetes could recall more accurately that their children were not supplemented with vitamin $\mathrm{D}$ in infancy, bias could result which would tend to inflate the association in favour of supplementation. Furthermore, none of the case-control studies included used an objective method to ascertain the vitamin D status of cases or controls, and none attempted to quantify the total amount of vitamin $\mathrm{D}$ intake from the diet or that from exposure to the sun. In addition, use of healthy controls without prior checking of non-diabetic status could lead to some misclassification. Case-control studies are also susceptible to bias because other risk factors of type 1 diabetes could be unbalanced across children who were supplemented and those who were not, with breastfeeding being an obvious example. Some of the included studies $^{20-2226}$ made an attempt to control for breastfeeding ( $<3$ months), but no information is given about volumes of formula milk consumed or amount of vitamin $\mathrm{D}$ in the formula milk. While the individual studies tried to control for a number

\section{What is already known on this subject}

Observational studies have suggested that vitamin D supplementation may reduce the risk of type 1 diabetes, but the results of the studies have been inconsistent.

\section{What this study adds}

- Meta-analysis of the results of observational studies suggest that the risk of type 1 diabetes is significantly reduced in those who were supplemented in childhood with vitamin $D$ compared to those who were not supplemented (odds ratio $0.71,95 \% \mathrm{Cl} 0.60$ to 0.84 ).

- Randomised controlled trials with long periods of follow-up are needed to establish causality and the best formulation, dose, duration and period of supplementation.

of potential confounding factors, it is possible that a number of other confounding factors could have been unbalanced across children who were supplemented and those who were not. Finally, we considered those who used cod liver oil as a supplement to be similar to those receiving other forms of vitamin $\mathrm{D}$. This could lead to erroneous conclusions as cod liver oil contains other components that could be protective against type 1 diabetes.

\section{CONCLUSION}

In conclusion, there is evidence from observational studies that vitamin D supplementation in infancy might be protective against the development of type 1 diabetes. Despite limitations, the Hill criteria for causality ${ }^{38}$ seem to be fulfilled. However, for concrete conclusions to be reached, adequately powered, randomised controlled trials with long periods of follow-up would be required to establish causality and the best formulation, dose, duration and period of supplementation.

\section{Funding: None.}

\section{Competing interests: None.}

Ethics approval: Not required.

Contributors: CSZ (guarantor) and AKA were both responsible for study selection, data extraction, validity assessment, data interpretation and writing the paper.

\section{REFERENCES}

1. Kimpimaki T, Kupila A, Hamalainen AM, et al. The first signs of beta-cell autoimmunity appear in infancy in genetically susceptible children from the general population: the Finnish Type 1 Diabetes Prediction and Prevention Study. J Clin Endocrinol Metab 2001;86:4782-8.

2. Kukreja A, Maclaren NK. Autoimmunity and diabetes. J Clin Endocrinol Metab 1999;84:4371-8.

3. Harris SS. Vitamin D in type 1 diabetes prevention. J Nutr 2005;135:323-5.

4. Gillespie KM. Type 1 diabetes: pathogenesis and prevention. CMAJ 2006;175:16570.

5. EURODIAB ACE Study Group. Variation and trends in incidence of childhood diabetes in Europe. Lancet 2000;355:873-6.

6. Onkamo P, Vaananen S, Karvonen M, et al. Worldwide increase in incidence of type I diabetes - the analysis of the data on published incidence trends. Diabetologia 1999;42:1395-403.

7. Risch N. Assessing the role of HLA-linked and unlinked determinants of disease. Am J Hum Genet 1987:40:1-14.

8. Kaprio J, Tuomilehto J, Koskenvuo M, et al. Concordance for type 1 (insulindependent) and type 2 (non-insulin-dependent) diabetes mellitus in a populationbased cohort of twins in Finland. Diabetologia 1992;35:1060-7. 
9. Hollis BW, Pittard WB, Reinhardt TA. Relationships among vitamin D, 25hydroxyvitamin $\mathrm{D}$, and vitamin D-binding protein concentrations in the plasma and milk of human subjects. J Clin Endocrinol Metab 1986;62:41-4.

10. Gartner LM, Greer FR, Section on Breastfeeding and Committee on Nutrition. Prevention of rickets and vitamin $\mathrm{D}$ deficiency: new guidelines for vitamin $\mathrm{D}$ intake. Pediatrics 2003;111:908-10.

11. Webb AR, Kline L, Holick MF. Influence of season and latitude on the cutaneous synthesis of vitamin D3: exposure to winter sunlight in Boston and Edmonton will not promote vitamin D3 synthesis in human skin. J Clin Endocrinol Metab 1988;67:373-8.

12. Hamlyn B, Brooker S, Oleinikova K, et al. Infant feeding 2000. London: HMSO, 2002.

13. Hypponen $\mathbf{E}$, Laara $\mathbf{E}$, Reunanen $\mathrm{A}$, et al. Intake of vitamin $\mathrm{D}$ and risk of type 1 diabetes: a birth-cohort study. Lancet 2001;358:1500-3.

14. Zipitis CS, Markides GA, Swann IL. Vitamin D deficiency: prevention or treatment? Arch Dis Child 2006;91:1011-14.

15. Merlino LA, Curtis J, Mikuls TR, et al. Vitamin D intake is inversely associated with rheumatoid arthritis: results from the lowa Women's Health Study. Arthritis Rheum 2004;50:72-7.

16. Hayes CE. Vitamin D: a natural inhibitor of multiple sclerosis. Proc Nutr Soc 2000;59:531-5.

17. Littorin B, Blom P, Scholin A, et al. Lower levels of plasma 25-hydroxyvitamin D among young adults at diagnosis of autoimmune type 1 diabetes compared with control subjects: results from the nationwide Diabetes Incidence Study in Sweden (DISS). Diabetologia 2006:49:2847-52.

18. Keen H, Ekoe JM. The geography of diabetes mellitus. Br Med Bull 1984;40:359-65.

19. Luong KV0, Hoang Nguyen LT, Pham Nguyen DN. The role of vitamin D in protecting type 1 diabetes mellitus. Diabetes Metab Res Rev 2005;21:338-46.

20. The EURODIAB Substudy 2 Study Group. Vitamin D supplement in early childhood and risk for type 1 (insulin-dependent) diabetes mellitus. Diabetologia 1999;42:51-4.

21. Stene LC, Ulriksen J, Magnus $P$, et al. Use of cod liver oil during pregnancy associated with lower risk of type I diabetes in the offspring. Diabetologia 2000;43:1093-8.

22. Stene LC, Joner G. Use of cod liver oil during the first year of life is associated with lower risk of childhood-onset type 1 diabetes: a large, population-based, case-control study. Am J Clin Nutr 2003;78:1128-34.
23. PHRU. Critical Appraisal Skills Programme. Oxford, UK: PHRU, 2007. http://www. phru.nhs.uk/Pages/PHD/CASP.htm (accessed 28 January 2008)

24. Clarke M, Oxman AD. Selecting studies. Cochrane reviewers' handbook 4.2.0. Oxford: Update Software, 2003.

25. Blettner M, Sauerbrei W, Schlehofer B, et al. Traditional reviews, metaanalyses and pooled analyses in epidemiology. Int J Epidemiol 1999;28:1-9

26. Tenconi MT, Devoti G, Comelli $M$, et al. Major childhood infectious diseases and other determinants associated with type 1 diabetes: a case-control study. Acta Diabetol 2007:44:14-19.

27. Mathieu C, Gysemans C, Giulietti A, et al. Vitamin D and diabetes. Diabetologia 2005; 48:1247-57.

28. Adorini L, Penna G, Giarratana N, et al. Inhibition of type 1 diabetes development by vitamin D receptor agonists. Curr Med Chem Anti-Inflammatory Anti-Allergy Agents 2005; 4:645-51

29. Marienfeld S, Hummel S, Ziegler A-G, et al. Infant nutrition and type 1 diabetes. Dtsch Arztebl Ausg A 2007;104:570-5.

30. Staeva-Vieira T, Peakman M, Von Herrath M. Translational mini-review series on type 1 diabetes: immune-based therapeutic approaches for type 1 diabetes. Clin Exp Immunol 2007;148:17-31

31. Kaupper T, Walter M, Ziegler A-G. Immunointervention in newly diagnosed type diabetes. Dtsch Med Wochenschr 2006;131:2783-8.

32. Harris $\mathbf{S}$. Can vitamin D supplementation in infancy prevent type 1 diabetes? Nutr Rev 2002:60:118-21.

33. Brekke HK, Ludvigsson J. Vitamin D supplementation and diabetes-related autoimmunity in the ABIS study. Pediatr Diabetes 2007:8:11-14.

34. Wicklow BA, Taback SP, Sanjeevi CB, et al. Feasibility of a type 1 diabetes primary prevention trial using $2000 \mathrm{IU}$ vitamin D3 in infants from the general population with increased HLA-associated risk. Ann N Y Acad Sci 2006;1079:310-12.

35. Zella JB, DeLuca HF Vitamin D and autoimmune diabetes. J Cell Biochem 2003;88:216-22.

36. Visalli $\mathbf{N}$, Sebastiani $\mathrm{L}$, Adorisio E, et al. Environmental risk factors for type 1 diabetes in Rome and province. Arch Dis Child 2003:88:695-8.

37. Norris JM. Can the sunshine vitamin shed light on type 1 diabetes? Lancet 2001;358:1476-8

38. Hill AB. The environment and disease: association or causation? Proc $R$ Soc Med 1965; 58:295-300

\section{BNF for Children 2007-Easing shared care prescribing}

Facilitating seamless care of children between hospital and the community Independent research shows that BNFC is improving the quality of prescribing for children under shared care arrangements between primary and secondary care.

For more information visit bnfc.org. To order go to www.pharmpress.com. 